\title{
Double left anterior descending artery: Congenital anomaly or normal variant of coronary arteries?
}

\author{
Michał Spałek ${ }^{1,2}$, Alicja Stępień-Wałek ${ }^{3}$, Joanna Paszkiewicz ${ }^{4}$, \\ Beata Wożakowska-Kapłon ${ }^{1,3}$
}

${ }^{1} J a n$ Kochanowski University, Faculty of Medicine and Health Sciences, Kielce, Poland

${ }^{2}$ Department of Diagnostic Imaging, Swietokrzyskie Oncology Center, Kielce, Poland

${ }^{3} 1^{\text {st }}$ Department of Cardiology and Electrotherapy, Swietokrzyskie Cardiology Center, Kielce, Poland

${ }^{4}$ Medical Imaging Department Doncaster and Bassetlaw Hospitals NHS Foundation Trust,

Doncaster, United Kingdom

A 73-year-old female patient was referred for coronary artery computed tomography angiography (CTA) due to dyslipidemia, hypertension, and mild ventricular arrhythmia. The study demonstrated a double left anterior descending artery (LAD) Type 1 variant (Fig. 1A) and significant atherosclerotic lesions in the coronary arteries. Conventional coronary angiography confirmed presence of an anomaly and significant atherosclerotic lesions within the second marginal branch ( $\mathrm{Mg} 2)$ and the right coronary artery (RCA). The patient was treated with percutaneous coronary intervention (PCI) of $\mathrm{Mg} 2$ and RCA with drug-eluting stenting (DES).
A 63-year-old female smoker with hypertension, had a positive stress test with low exercise tolerance (5.9 METS) and significant ST depression in leads II, III, aVF, V5 and V6, with chest pain. The 24-h Holter electrocardiography monitoring demonstrated ventricular arrhythmia with nonsustained ventricular tachycardia (nsVT). The CTA revealed multifocal significant atherosclerotic lesions and dual LAD Type 1 (Fig. 1B). The patient did not consent to coronary angiography.

Computed tomography angiography is the preferred imaging method in the assessment of coronary arteries anomalies. The main advantages

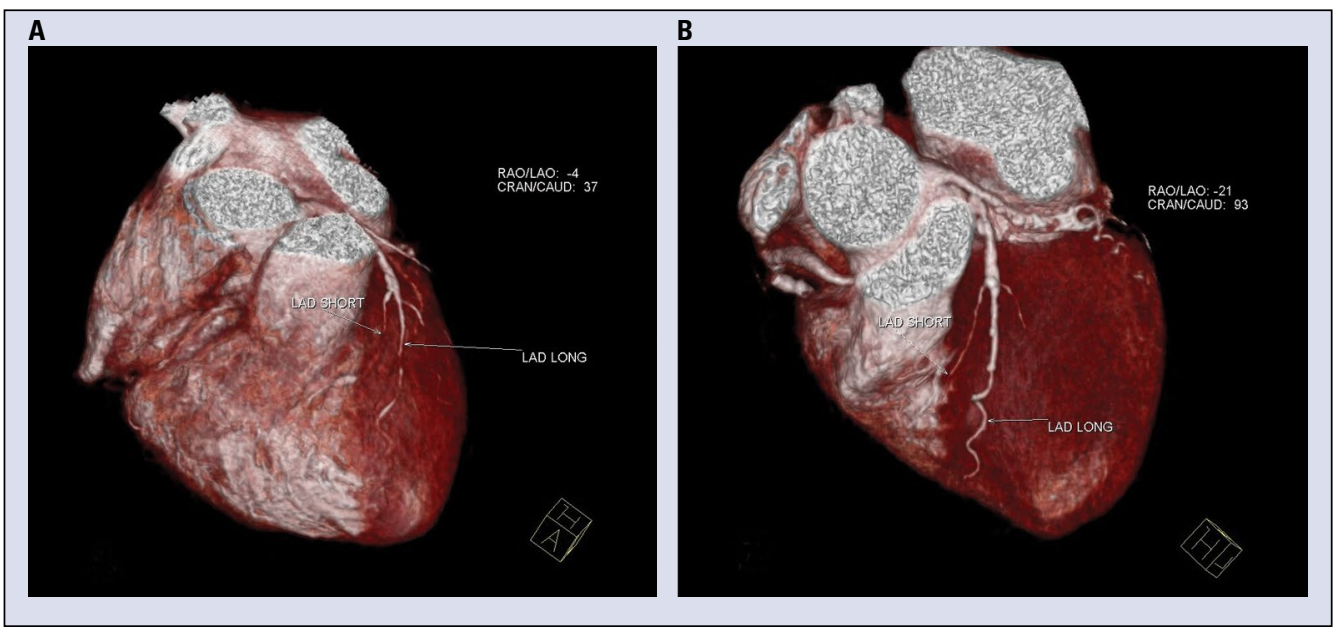

Figure 1. Double left anterior descending artery (LAD) Type 1. in computed tomography examination, volume rendering technique; A. Patient 1; B. Patient 2.

Address for correspondence: Dr. Michał Spałek, Jan Kochanowski University, Faculty of Medicine and Health Sciences, Aleja IX Wieków Kielc 19, 25-900 Kielce, Poland, e-mail: michal_spa@op.pl

Received: 29.06.2016 Accepted: 13.11.2016 
of coronary CTA include a high resolution and 3-dimensional reconstructions in any plane, which facilitates the evaluation of anatomy and the degree of athermanous stenosis. It is also useful in planning an invasive procedure.

Cardiac CTA is recommended by the European Society of Cardiology as the imaging method of choice in the evaluation of various coronary artery anomalies.

Congenital anomalies of coronary arteries are relatively rare. The frequency of occurrence in the human population is from $0.6 \%$ to $1.2 \%$ [1]. Clinical manifestation depends on the type of anomaly, most of which are clinically insignificant and are incidental.

One of the anomalies is dual LAD, where at least two different coronary arterial branches descend in the anterior interventricular groove. This is a mild and most often clinically silent anomaly, however recognizing this anomaly becomes important when planning an invasive treatment.

The initial classification of coronary artery variations were based on coronary angiography of 2140 patients describing four types of those as it was in Spindola-Franco publication in 1983 [2].

Constant development of non-invasive imaging methods such as CT, helps to identify undocumented types. The fifth type of dual LAD was described in 2010 by Manchanda et al. [3], Type 6 in 2012 by Maroney et al. [4] and the final three types (Type 7, Type 8, and Type 9) were described in 2015 by Bozlar et al. [5] who investigated 1337 patients using CTA and found $56(4.2 \%)$ cases of double LAD. The most common is Type 1 of double LAD (3.59\%). Types 5, 6 and 7 of double LAD can potentially cause sudden cardiac death.

Taking into account the above mentioned reports and the occurring frequency of various types of double LAD subject for discussion was warrented as to whether Type 1 of double LAD should be regarded as a variance and the remaining types classified as anomalies.

\section{Conflict of interest: None declared}

\section{References}

1. Angelini P, Velasco JA, Flamm S. Coronary anomalies: incidence, pathophysiology, and clinical relevance. Circulation. 2002; 105(20): 2449-2454, indexed in Pubmed: 12021235.

2. Spindola-Franco H, Grose R, Solomon N. Dual left anterior descending coronary artery: Angiographic description of important variants and surgical implications. American Heart Journal. 1983; 105(3): 445-455, doi: 10.1016/0002-8703(83)90363-0.

3. Manchanda A, Qureshi A, Brofferio A, et al. Novel variant of dual left anterior descending coronary artery. J Cardiovasc Comput Tomogr. 2010; 4(2): 139-141, doi: 10.1016/j.jcct.2009.12.007, indexed in Pubmed: 20430346.

4. Maroney J, Klein LW. Report of a new anomaly of the left anterior descending artery: type VI dual LAD. Catheter Cardiovasc Interv. 2012; 80(4): 626-629, doi: 10.1002/ccd.23219, indexed in Pubmed: 21953811.

5. Bozlar U, Uğurel MŞ, Sarı S, et al. Prevalence of dual left anterior descending artery variations in CT angiography. Diagn Interv Radiol. 2015; 21(1): 34-41, doi: 10.5152/dir.2014.14275, indexed in Pubmed: 25333217. 